\title{
Intraoperative Epicoronary Ultra-High Frequency Ultrasound in Coronary Artery Bypass Grafting Surgery
}

\author{
Ashley Fritz ${ }^{1}$, Archer Martin ${ }^{1}$, Erol Belli ${ }^{2}$, and Steven Clendenen ${ }^{1}$ \\ ${ }^{1}$ Mayo Clinic \\ ${ }^{2}$ University of South Florida
}

June 8, 2020

\begin{abstract}
The use of intraoperative epicardial ultrasound in order to aid physicians and surgeons in open cardiac surgery has been established for quite some time. Recently, the development of ultra-high frequency ultrasound (UFHUS) technology has resulted in high-resolution imaging capabilities previously unavailable for clinical use. This report is the first to describe the use of intraoperative UFHUS epicoronary scanning to assess coronary anatomy and visualize cardioplegia flow within native coronary vessels.
\end{abstract}

\section{Case Report:}

The use of intraoperative epicardial ultrasound to scan coronary arteries in order to assess bypass graft targets and survey efficacy of surgical re-vascularization is well described within the literature.(1) Whereas the international Registry for Quality Assessment with Ultrasound Imaging and TTFM in Cardiac Bypass Surgery (REQUEST) trial utilized a $15 \mathrm{Mhz}$ probe for scanning, recent literature describes the use of an ultrahigh frequency ultrasound (UHFUS) $70 \mathrm{Mhz}$ probe for anatomical assessment during non-cardiac surgery.(1, 2) We present a case of intraoperative UHFUS use for epicoronary scanning in a patient undergoing coronary artery bypass grafting $(\mathrm{CABG})$ surgery. The patient gave written permission to publish this case.

A 64 year old male with a past medical history significant for hypertension and hyperlipidemia was referred to our service for evaluation of his coronary artery disease. He reported worsening chest pain with activity over the year prior to referral, and was evaluated pre-operatively with both non-invasive and invasive cardiac testing. Transthoracic echocardiography showed concentric left ventricular hypertrophy with intact biventricular function. Left heart catheterization showed extensive coronary disease including $95 \%$ left main coronary occlusion, multiple discrete left anterior descending (LAD) coronary occlusions ranging from $50 \%$ $100 \%$, and a $90 \%$ right coronary artery (RCA) lesion. The patient was scheduled for an on-pump CABG surgery.

The patient was brought to the operating room where he was placed under general anesthesia. After median sternotomy was performed, harvesting of saphenous vein and bilateral mammary arteries commenced without event. Both antegrade and retrograde cardioplegia were used to ensure electromechanical silence of the heart during cardiopulmonary bypass. A $70 \mathrm{Mhz}$ UFHUS probe was employed to assess the surface anatomy of the native, diseased coronary arteries in order to optimize anatomical planning for graft targets. After being placed in a sterile sleeve, the probe was used to assess the LAD and posterior descending artery (PDA). As noted in figure 1 and video 1, both cardioplegia flow and significant calcifications were seen in the PDA. Figure 2 shows the LAD in short axis, and calcifications are noted for both their bright echogenicity and ultrasonic dropout artifact. Coronary grafting commenced and a Doppler flowmeter was used to confirm 
patency. The patient had a successful separation from cardiopulmonary bypass, uneventful post-operative course, and was discharged on post-operative day seven.

\section{Discussion:}

The REQUEST trial reported that the use of high-frequency ultrasound (HFUS) is not only helpful for evaluating coronary targets, but that physicians should consider incorporating it into their usual practice for CABG surgery.(1) Higher frequency probes (55 Mhz) have been used to examine temporal arteries in the setting of Giant Cell disease, with greater resolution allowing for accurate assessment of intima layer thickness that correlated with histological diagnosis.(3)

As the probe frequency increases, image resolution improves while sound penetration decreases.(2) Therefore, the use of UFHUS for epicoronary scanning presents a theoretical benefit over lower-frequency probes, yet no comparison of image quality was performed during this case. While our probe was rated up to 70 Mhz frequency, a frequency of $50 \mathrm{Mhz}$ was used in order to achieve appropriate ultrasound penetration depth of $5.5 \mathrm{~mm}$ for epicoronary scanning. In our case, the UFHUS allowed for real-time evaluation of the extensive native coronary calcification during cardiopulmonary bypass. It can often be challenging to assess this in an arrested heart, and inadvertent selection of a distal graft site with heavy calcification requiring endarterectomy may lead to lower long-term patency. In addition to serving as an imaging aid for selection of acceptable graft target sites, other potential applications of UFHUS epicoronary scanning include confirming cardioplegia flow in the setting of low coronary sinus pressures and appraising distal coronary flow post-revascularization.

To our knowledge, this is the first report of the use of UFHUS for epicoronary scanning in CABG surgery. Further study is necessary to compare the efficacy of UFHUS versus high frequency ultrasound for use in epicoronary scanning during CABG surgery.

\section{Figure Legend:}

Figure 1: Short axis view of the posterior descending artery. Note the significant calcifications and subsequent acoustic shadowing. A branch of the middle cardiac vein is present on the right side of the picture.

Video 1: This video shows the presence of retrograde cardioplegia flow within the cardiac arterial and venous system during cardiopulmonary bypass.

Figure 2: Short axis view of the left anterior descending artery. The arrows points to the presence of calcification. Additionally, intraluminal irregularities are noted within the artery.

\section{References:}

1. Taggart DP, Thuijs D, Di Giammarco G, Puskas JD, Wendt D, Trachiotis GD, et al. Intraoperative transit-time flow measurement and high-frequency ultrasound assessment in coronary artery bypass grafting. J Thorac Cardiovasc Surg. 2019.

2. Boczar D, Forte AJ, Oliver JD, McClain RL, Murray P, Clendenen SR. Intraoperative evaluation of cervical nerve root avulsion using ultra-high-frequency ultrasound system. Case Reports Plast Surg Hand Surg. 2019;6(1):43-6.

3. Sundholm JKM, Paetau A, Alback A, Pettersson T, Sarkola T. Non-Invasive Vascular Very-High Resolution Ultrasound to Quantify Artery Intima Layer Thickness: Validation of the Four-Line Pattern. Ultrasound Med Biol. 2019;45(8):2010-8. 

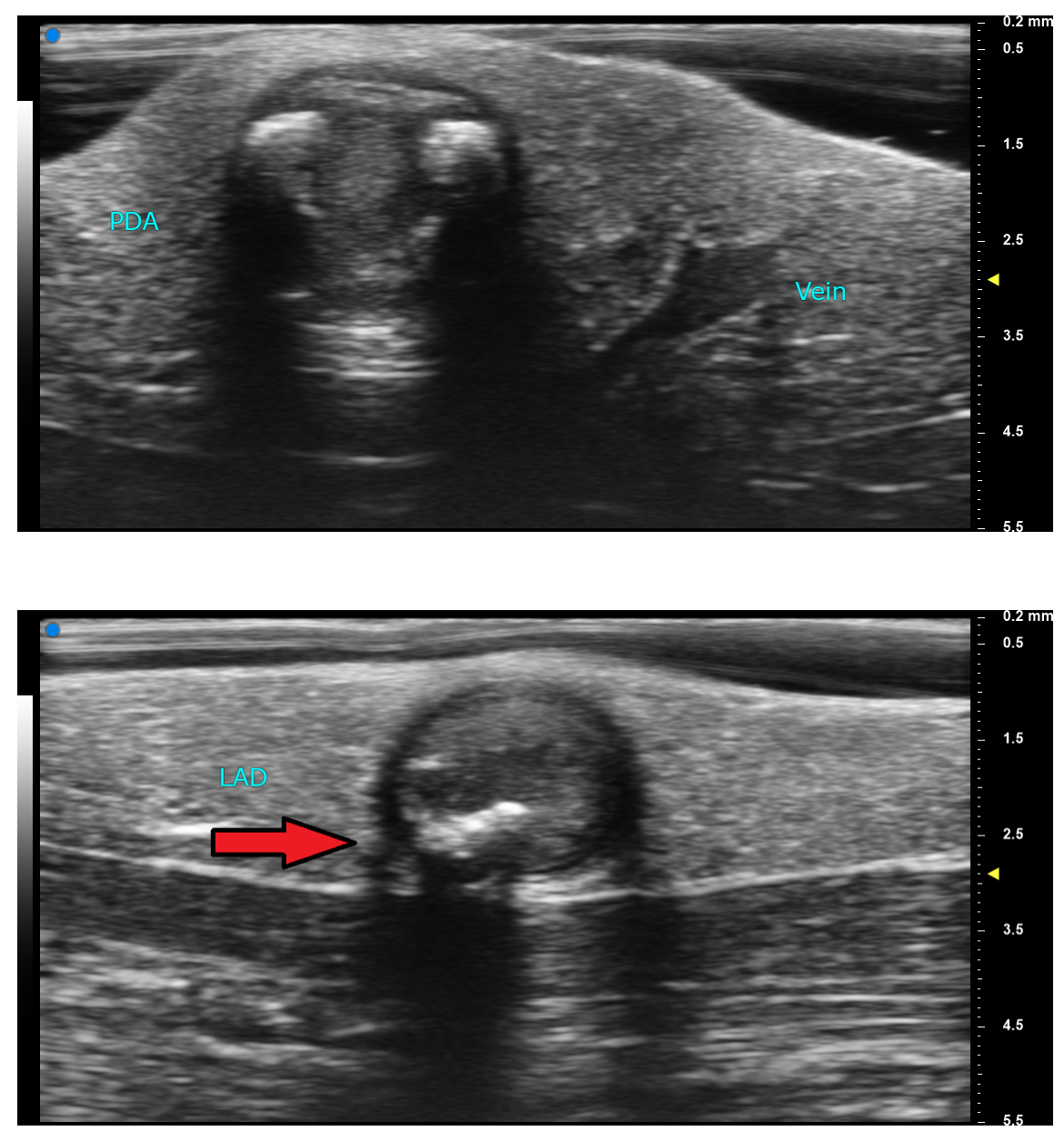

\section{Hosted file}

Video 1.mov available at https://authorea.com/users/330544/articles/457294-intraoperativeepicoronary-ultra-high-frequency-ultrasound-in-coronary-artery-bypass-grafting-surgery 